DOI: 10.5604/01.3001.0013.2538

\title{
OPPORTUNITIES FOR THE OUT OF THE 1550 nm WINDOW TRANSMISSION
}

\author{
Jarosław Piotr Turkiewicz ${ }^{1,2}$ \\ ${ }^{1}$ Warsaw University of Technology, Faculty of Electronics and Information Technology, Warsaw, Poland, ${ }^{2}$ Orange Labs Poland, 7 Obrzeżna, Warsaw
}

Abstract. In this paper, opportunities for transmission in the $850 \mathrm{~nm}$ and $1310 \mathrm{~nm}$ windows are reviewed. In particular, the mentioned windows can be utilized for the data centre related transmission.

Keywords: optical communication, optical fibre, optical amplifier

\section{MOŻLIWOŚCI WYKORZYSTANIA TRANSMISJI POZA PASMEM 1550 nm}

Streszczenie. W artykule dokonano analizy możliwości wykorzystania okien transmisyjnych $850 \mathrm{~nm}$ i $1310 \mathrm{~nm}$. Rozważane okna można wykorzystać do realizacji transmisji dla potrzeb centrów danych.

Słowa kluczowe: komunikacja optyczna, światłowód, wzmacniacz optyczny

\section{Introduction}

The ever growing number of information and communication network users like Internet, development of bandwidth hungry applications, e.g. high quality video streaming as well as increased quality requirements, e.g. low latency is driving development of the telecommunication networks. The optical fibre is the only one physical medium that can provide high transmission capacity, while maintaining very long transmission distances, up to trans continental ones. The importance of the optical fibre to the development of civilization, has been recognized by awarding Charles K. Kao in 2009 Nobel Prize in physics for "for ground breaking achievements concerning the transmission of light in fibres for optical communication" [4].

The potential of optical fibre transmission had been very quickly recognized and it has been widely adopted for the signal transmission. For the very long time, the key application area was high capacity (whatever did it mean at the given moment in time) over long distances, where the high cost of the transmission system was shared among many users. Recently, we observe rapid increase of data rates used in the computer inert and intra board communication as well as growth of the data storage and processing centres. In such application areas, copper cable transmission cannot handle high data rates transmission over the required distances. However, such demand can be fulfilled by the optical fibre transmission. One could say, that sarcastically the optical fibre transmission is covering shorter and shorter distances.

Due to the lowest attenuation of $0.2 \mathrm{~dB} / \mathrm{km}$ and excellent amplification technology, namely erbium doped fibre amplifier (EDFA) the $1550 \mathrm{~nm}$ transmission window is a window of choice to realize optical fibre transmission. The other transmission windows like $850 \mathrm{~nm}$ or $1310 \mathrm{~nm}$ seemed to be until recently almost entirely forgotten. However, the rise of new application areas like interand intra-data centre transmission that put attention on the features like low cost and high energy efficiency, give prospect for efficient utilization of other transmission windows.

The high data rate ultrashort data links, called data interconnect, currently operate at the data rates $25 \mathrm{Gbit} / \mathrm{s}$ with the foreseen increase to $50 \mathrm{Gbit} / \mathrm{s}$ and even $100 \mathrm{Gbit} / \mathrm{s}$ over the distances up to a few hundred meters to cover the data centre area. The key requirements of the data interconnects are small footprint, limited energy consumption and overall low component cost. Such data interconnects can be realized in the $850 \mathrm{~nm}$ transmission window. The $1310 \mathrm{~nm}$ transmission window can be used to realize high data rate transmission over the distances up to few dozen kilometres, leveraging a key standard single mode fibre (SSMF) advantage at $1310 \mathrm{~nm}$, namely low value of chromatic dispersion, which translates into the simplified system design (lack of dispersion compensation) as well as straightforward system installation, which is one of the most sought features of the data communication equipment. Table 1 shows comparison of the key transmission window characteristics.
Table 1. Optical fibre transmission window comparison

\begin{tabular}{|l|l|l|l|}
\hline & \multicolumn{1}{|c|}{$1550 \mathrm{~nm}$} & \multicolumn{1}{c|}{$1310 \mathrm{~nm}$} & \multicolumn{1}{c|}{$850 \mathrm{~nm}$} \\
\hline$\lambda$ number & Over 100 & Up to several & A few \\
\hline Single data rate & $400 \mathrm{Gbit} / \mathrm{s}$ & $50 \mathrm{Gbit} / \mathrm{s}$ & $25 \mathrm{Gbit} / \mathrm{s}$ \\
\hline Range & $>1000 \mathrm{~km}$ & $<50 \mathrm{~km}$ & $<1 \mathrm{~km}$ \\
\hline Optical amplifier & EDFA & SOA & none \\
\hline Total capacity & Tbit/s & $100 \mathrm{Gbit} / \mathrm{s}$ & $100 \mathrm{Gbit} / \mathrm{s}$ \\
\hline Fibre & SSMF & SSMF & MM \\
\hline Attenuation & $0.2 \mathrm{~dB} / \mathrm{km}$ & $0.4 \mathrm{~dB} / \mathrm{km}$ & $4 \mathrm{~dB} / \mathrm{km}$ \\
\hline
\end{tabular}

\section{1. $850 \mathrm{~nm}$ data interconnects}

The fibre of choice for the $850 \mathrm{~nm}$ window is a multi-mode fibre (MMF). Except the high attenuation at $850 \mathrm{~nm}$ around $4 \mathrm{~dB} / \mathrm{km}$, the multi-mode fibres transmission is affected by the modal and chromatic dispersion, which limits the available transmission bandwidth to about $4.7 \mathrm{GHz} \cdot \mathrm{km}$ for the newest generation of MMF. However, for the ultra-short links, e.g. of $100 \mathrm{~m}$, the fibre bandwidth increases to $47 \mathrm{GHz}$ and even higher for meter range distances. Further, the core dimeter of MMF fibres is $50 \mu \mathrm{m}$. Such large core diameter allows easy coupling with the emitting lasers and receiving photo-detectors.

The vertical-cavity emitting lasers (VCSELs) at $850 \mathrm{~nm}$ are characterized by surface light emission, the modulation bandwidth up to $25 \mathrm{GHz}$, coupled output power of a few milliwatt and driving current of a few miliamper [5]. Figure 1 shows the spectrum and light-intensity-voltage characteristics of the $850 \mathrm{~nm}$ VCSEL. The high modulation bandwidth and low energy consumption, while maintaining desired optical power allows realization of the cost-effective transmission systems with a few dozen Gbit/s data rate over up to a few hundred meter distances based on MMF and VCSEL [19]. The system simplicity (direct modulation and detection) and limited power consumption translate into low transmission cost, which is a key feature required for the data interconnects, which number can exceed a few dozen thousand in a single data centre.
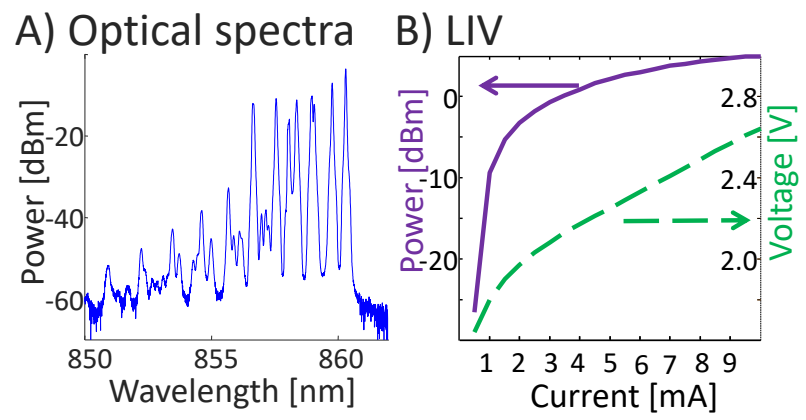

Fig. 1. $850 \mathrm{~nm}$ VCSEL spectral and LIV characteristics 
Figure 2 shows the exemplary eye diagram of the $850 \mathrm{~nm}$ VCSEL operated at various data rates for the amplitude binary on-off keying (OOK) modulation. Up to the $35 \mathrm{Gbit} / \mathrm{s}$ the yes is almost not distorted, while distortions can be observed for higher data rates. The data rate related distortions are dueto the limited bandwidth of the utilized components like VCSEL and photoreceiver, which show bandwidth of about $22 \mathrm{GHz}$. Nevertheless, as we can see up to the data rate of $50 \mathrm{Gbit} / \mathrm{s}$ the eye diagram is widely open in the middle, indicating proper transmission and signal reception. The shown eye diagram, were captured in so called back-to-back (b2b) configuration, i.e. directly connecting transmitter to the receiver, as such the transmission distance was in the range of a few meters as the length of the pigtails attached to the components. It is important to note, that such a distance perfectly cover the distance required to connect components within a 19" standard rack of height up to $2 \mathrm{~m}$.
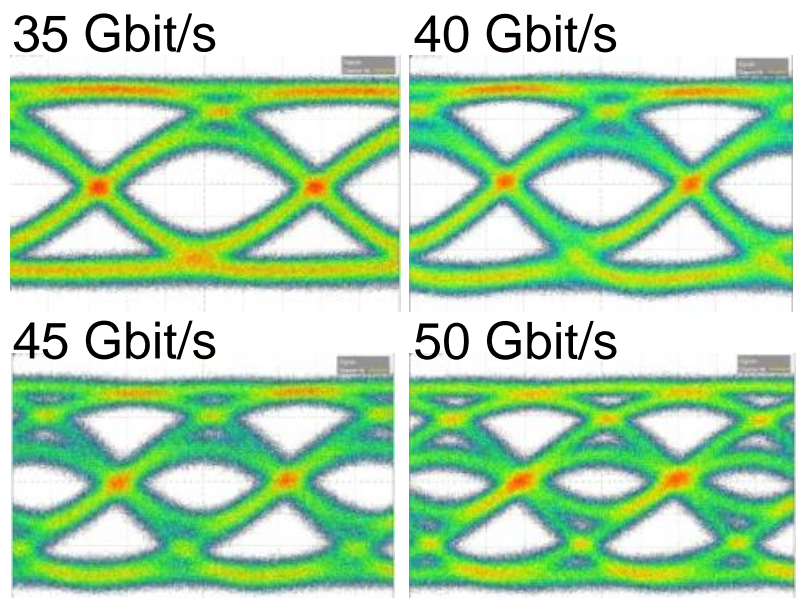

Fig. 2. $850 \mathrm{~nm}$ VCSEL eye diagrams at various data rates

Data interconnect transmission capabilities, in term of the achievable data rate will not only be affect by the limited bandwidth of the VCSEL and photo-detectors but more importantly as the distance grow by transmission properties of the MMF.

To overcome limitations related to the MMF chromatic dispersion, single mode (SM) VCSELs have been developed [7]. The single mode VCSELs are characterized by the limited optical spectrum width, since preferably only one wavelength mode occurs. In such a way, the influence of the chromatic dispersion is significantly limited. SM VCSEL offer potential to cover much higher distances that the multi mode (MM) VCSELs, which is critical to cover inter-rack/room/building distances in the data centre, without switching to another more complex and expensive transmission technology. Obviously, the SM VCSEL modulation bandwidth must stay in the range of a few dozen $\mathrm{GHz}$

$\mathrm{MM}$
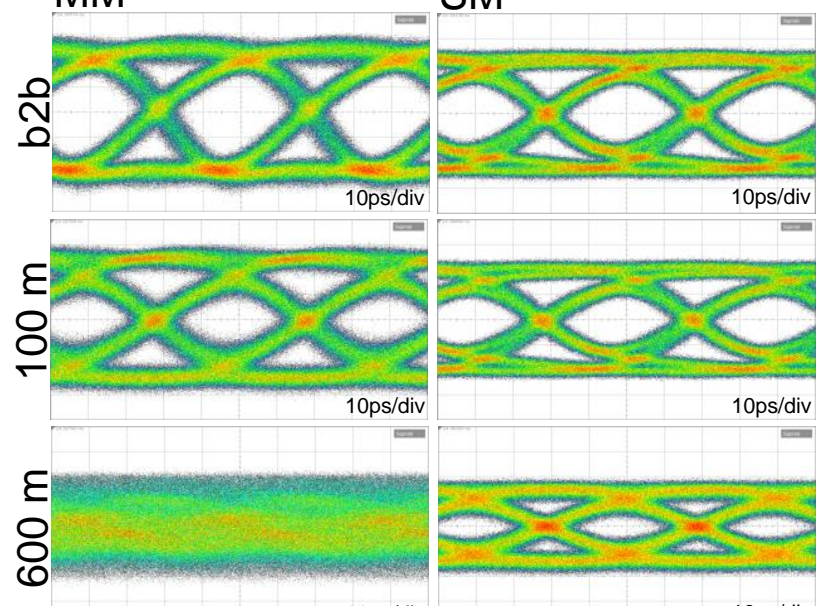

$10 \mathrm{ps} / \mathrm{div}$

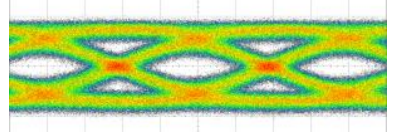

$10 \mathrm{ps} / \mathrm{div}$
Figure 3 presents the eye diagrams at $25 \mathrm{Gbit} / \mathrm{s}$ captured at the various MMF lengths with MM and SM VCSELs. As we can see for $\mathrm{b} 2 \mathrm{~b}$ configuration (a few meter transmission) both SM and MM VCSEL signal are basically the same and show excellent system operation. With the increased transmission distance the signal distortion become visible. For the $100 \mathrm{~m}$ transmission, the MM VCSEL eye diagram is slightly closed compared to b2b, while at 600 it is completely distorted. For the SM VCSEL the eye diagram remains widely open up to $600 \mathrm{~m}$, proofing that much longer transmission distances can be bridged with such type of VCSELS.

The realized transmission systems with MM VCSELs included transmission up to $54 \mathrm{Gbit} / \mathrm{s}$ with the OOK modulation over the distances up to $1 \mathrm{~km} \mathrm{[1]} \mathrm{and} 2.4 \mathrm{~km}$ [17]. Further, the MM and SM VCSEL can be utilized in transmission with advanced modulation formats. Advanced modulation formats, in opposition to the binary modulation, allow to transit more than one bit in one symbol, e.g. 2 bit/s for four level pulse-amplitude modulation (PAM4) modulation. Therefor the spectrum utilization is significantly improved from $1 \mathrm{bit} / \mathrm{s} / \mathrm{Hz}$ to 2 and more bit/s/Hz. That allows to overcome bandwidth limitations of the existing components and increase the achievable data rates. The drawback of the proposed solution is more complex structure of the transmitter and receiver. Further the required, signal-to-noise ratio is much higher than for binary modulation.

The performed work on the advanced modulation format transmission includes transmission with the PAM-4 modulation, again showing superior SM over MM VCSEL performance [16]. Even higher spectral efficiency was achieved with the carrier less amplitude-phase (CAP) modulation, which was utilized in many application with the very limited system bandwidth [25]. A variant of CAP modulation, namely multi-CAP was used in [14] to demonstrate the record at the time of publishing transmission of $107 \mathrm{Gbit} / \mathrm{s}$. In multi-CAP transmission, the system bandwidth is divided into the sub-bands, in which one of the individual CAP signals are transmitted with the highest possible modulation order. In such a way, sub-bands with the excellent transmission properties transmit signals with high data rate, while the sub-bands with the limited transmission performance are utilized for the lower data rate signals. Further, the sub-band can be turned on-off adjusting to the varying traffic and therefore the variable data rate and energy efficient transmission can be realized [13].

To allow characterization and in general work with the newest VCSEL generations, a probe station had been designed and build. The probe station is based on the micrometric XYZ stages and allow connection of the electrical signals through the high bandwidth electrical probe as well as couple the optical signal into the fibre. The VCSEL chips can be observe though the side cameras. That simplifies the systems connections and adjustment. On Fig. 4., the photograph of the probe station is shown. The developed probe station can be used not only for the VCSEL testing but also for testing of other components like photo-detectors or even electronics circuits.

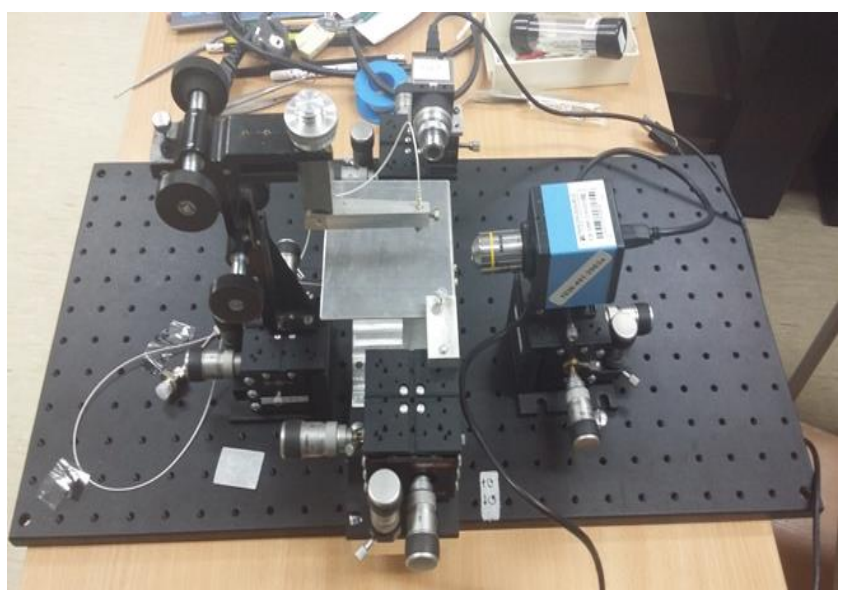

Fig. 4. Probe station used to test the bare (chip level) VCSELs 


\section{2. $1310 \mathrm{~nm}$ transmission}

The $1310 \mathrm{~nm}$ transmission window $(1260 \mathrm{~nm}-1360 \mathrm{~nm})$ was considered in the early years of optical fibre communication, namely mid 1970 s as the key transmission band. That is reflected in the alternative $1310 \mathrm{~nm}$ window name O-band, where $\mathrm{O}$ stands for original. Fibre technology improvements resulted in conquering other bands like C-band $(1550 \mathrm{~nm})$, where $\mathrm{C}$ stand for conventional. For the very long time, the $1310 \mathrm{~nm}$ band has been utilized just for the upstream traffic in the passive optical networks (PON). In PON networks the downstream traffic to the user is realized in the $1550 \mathrm{~nm}$ band. Such wavelength band division comes from availability of the low cost and low loss 1310/1550 nm band splitters and combiners. The downstream PON data rates are up to $10 \mathrm{Gbit} / \mathrm{s}$. In mid 2000s, work on the $100 \mathrm{G}$ and more Ethernet standard started, where one of the solutions is to transmit $100 \mathrm{G}$ and 400G Ethernet streams using multi-wavelength technology in the $1310 \mathrm{~nm}$ window. That is still bellow multi-terabit/s capacity of the $1550 \mathrm{~nm}$ band.

The key features of the $1310 \mathrm{~nm}$ transmission window are attenuation of about $0.3 \mathrm{~dB} / \mathrm{km}-0.4 \mathrm{~dB} / \mathrm{km}$, presence of the zerodispersion wavelength and therefore limited chromatic dispersion as well as pronounced presence of the non-linear effects due to the low dispersion value. The higher than in the $1550 \mathrm{~nm}$ wavelength band losses $(0.2 \mathrm{~dB} / \mathrm{km})$ must be compensated otherwise only very short unrepeated transmission can be realized. Three amplification technologies have been demonstrated for applications in the $1310 \mathrm{~nm}$ domain, namely semiconductor optical amplifier (SOA), praseodymium doped fibre amplifier (PDFA) and Raman amplifier. Recently, a bismuth doped fibre amplifier with the $25 \mathrm{~dB}$ gain, $3 \mathrm{~dB}$ saturation power of about $15 \mathrm{dBm}$ and noise figure (NF) of about $5 \mathrm{~dB}$ has been demonstrated [18]. SOA and PDFA demonstrate moderate gain, high noise figure and moderate saturation power [2]. Progress in the high power quantum dot lasers allows realization of the $1310 \mathrm{~nm}$ Raman amplifier with the gain over 15-18 $\mathrm{dB}$ and very low noise figure [3, 12]. The $1310 \mathrm{~nm}$ Raman amplifier has been tested in the various transmission experiments, e.g. [10] outperforming SOA. Table 2 summarizes key transmission properties of the $1310 \mathrm{~nm}$ window amplifier technologies.

Table 2. $1310 \mathrm{~nm}$ optical amplifier comparison

\begin{tabular}{|l|c|c|c|c|}
\hline & SOA & PDFA & Raman & BiDFA \\
\hline Gain $[\mathrm{dB}]$ & 25 & 25 & 18 & 25 \\
\hline Psat $[\mathrm{dBm}]$ & 10 & 10 & 15 & 15 \\
\hline NF $[\mathrm{dB}]$ & $>6$ & $>6$ & 4 & 5 \\
\hline
\end{tabular}

Chromatic dispersion affects transmitted signals by limiting the available transmission range for the given data rate or limiting the data rate for the given transmission distance. The chromatic dispersion limits for SSMF are specified in ITU-T Recommendation G.652 [15]. The zero dispersion wavelength, a wavelength where no chromatic dispersion occurs must be between $1300 \mathrm{~nm}$ and $1324 \mathrm{~nm}$. Figure 5 shows the chromatic dispersion limits for SSMF as specified in the ITU-T Recommendation G.652. The very low value of chromatic dispersion allows realization of the transmission systems without any form of the chromatic dispersion compensation. That just not only simplifies system design, but more importantly makes the installation straightforward, without necessity of dispersion measurements and compensation. These features are highly desirable in the data centre environment, where a large number of such systems must be installed. Further, the limited influence of the chromatic dispersion is a decisive advantage for the analog radio-over-fibre systems [6], where the signals are transmitted in the fibre in such a form than they can be directly emitted by the radio antenna just after the optical-toelectrical conversion. Such systems are of great importance for the development of the newest generation of mobile networks, namely $5 \mathrm{G}$, in particular for the ultra-high carrier frequencies, which are needed to realize ultra-broadband and therefore high data rate radio transmission. The targeted here data rates are in the range of a few Gbit/s to the user.

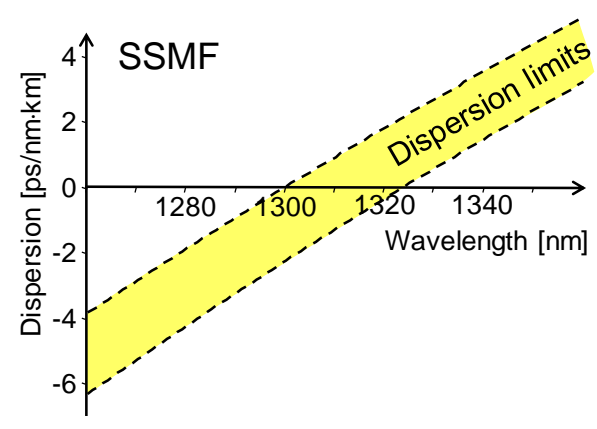

Fig. 5. SSMF chromatic dispersion limits at $1310 \mathrm{~nm}$

However, looking at the Fig. 5. we can notice that at the edges of the O-band, namely $1260 \mathrm{~nm}$ and $1360 \mathrm{~nm}$ chromatic dispersion of a few $\mathrm{ps} / \mathrm{nm} \cdot \mathrm{km}$ can be expected. Such high value of dispersion can influence high data rate transmission of $50 \mathrm{Gbit} / \mathrm{s}$ and more, even for distances of a few dozen kilometres. That dispersion value ca not be neglected and can be a source of severe performance limitations that can be omitted by the chromatic dispersion management in the fibre infrastructure [24].

Low value of chromatic dispersion can lead to pronounced nonlinear effect interactions like cross-phase modulation and in particular four-wave mixing (FWM). In FWM effect three co-propagating wave interacts with each other and a new wave is generated. A new FWM wave can appear at the data signal frequency, which will be a source of cross-talk. FWM can be effectively suppressed by lowering the signal power, transmission in the region with non-zero chromatic dispersion as well as large channel spacing. The conducted studies have shown that the channels spacing of about $250 \mathrm{GHz}$ allows to effectively suppress FWM, while maintaining relatively high signal power in the range of $0 \mathrm{dBm}$ for the $1310 \mathrm{~nm}$ band dense wavelength division multiplexed systems [8]. Obviously, spectral efficiency is not that high as in the $1550 \mathrm{~nm}$ band with the standard channels spacing of $50 \mathrm{GHz}$, nevertheless it is sufficient to realize transmission of a several and even few dozen channel with overall capacity in the order of Tbit/s.

Several research works have been devoted to that topic. In [20] $\mathrm{n} \times 25 \mathrm{Gbit} / \mathrm{s}$ transmission in the $1310 \mathrm{~nm}$ band has been investigated, while [21] demonstrates up to $400 \mathrm{Gbit} / \mathrm{s}$ transmission with eight wavelength channels and data rates of $40 \mathrm{Gbit} / \mathrm{s}$ and $50 \mathrm{Gbit} / \mathrm{s}$. Further, a single data channel transmission at $112 \mathrm{Gbit} / \mathrm{s}$ has been demonstrated in [9]. The polarization and wavelength multiplexing concept has been further expanded towards $1 \mathrm{Tbit} / \mathrm{s}$ transmission in [11].

One of the features of the $1310 \mathrm{~nm}$ band is feasibility of the parallel to the $1550 \mathrm{~nm}$ band utilization. The capacity of the optical fibre can be increased by the multi core or a few mode transmission. In such special fibre new spatial channels (multiple cores and/or multiple modes) are created. Obviously, the inter core and inter mode cross-talks must be compensated to achieve desired performance. Recognized alternative to that is utilization of the parallel to the $1550 \mathrm{~nm}$ wavelength bands like the $1310 \mathrm{~nm}$ or $1650 \mathrm{~nm}$ (U-band) [26]. That solution has advantage that the already existing fibre infrastructure can be used to carry additional data channels, postponing or even omitting necessity of the very expensive new fibre installation. Obviously, appropriate band multiplexers and demultiplexers must be inserted into the transmission line as well as suitable amplification technology must be used, with the most promising candidates of BiDFa and Raman amplifier. Due to the development of the all-optical signal processing techniques, the $1310 \mathrm{~nm}$ signals can be all-optically without any optical-electrical-optical conversion converted into the $1550 \mathrm{~nm}$ wavelength domain. Such an ultra-wide data wavelength conversion utilizing non-linear polarization rotation in the semiconductor optical amplifier has been demonstrated in [22]. In such a way, transparent all-bands optical networks can be realized. In other applications, the $1310 \mathrm{~nm}$ components were used for $1550 \mathrm{~nm}$ signal processing [20]. 


\section{Conclusions}

Optical fibre transmission technologies arc conquering new application areas. In particular, the growth of optical transmission is observed in the data center infrastructure. The deceive advantages of the optical fibre transmission are ultra-high data rates and energy efficiency, which cannot be fulfilled by the metal wire techniques. New transmission solution are tailored to the application needs. Here, the window of opportunity for the unutilized so far band has opened. The $850 \mathrm{~nm}$ window can be successfully applied to realize high data rate transmission at the ultra-short distances utilizing VCSELs and MMF. The $1310 \mathrm{~nm}$ window can be used to support intra data center transmission of high capacity over distances up to a few dozen kilometer.

\section{Acknowledgements}

The presented research has received funding from the European Union's Seventh Framework Programme (FP7/2007-2013) under grant agreement $n^{\circ} 619197$ - the ADDAPT project, Polish Ministry of Science and Higher Education science funds for years 2014-2017 granted for international project execution as well as the Polish National Science Centre NCN under the contract UMO2011/03/D/ST7/02497. Further, I would like to thank VI Systems, Berlin, Germany (v-i-systems.com) for cooperation on the $850 \mathrm{~nm}$ VCSEL transmission.

\section{References}

[1] Chi K.-L., Shi Y.-X., Chen X.-N., Chen J., Yang Y.-J., Kropp J.-R., Ledentsov Jr. N., Agustin M., Ledentsov N.N., Stepniak G., Turkiewicz J.P., Shi J.-W: Single-Mode 850-nm VCSELs for 54-Gb/s ON-OFF Keying Transmission Over 1-km Multi-Mode Fiber. IEEE Photonics Technology Letters 12/2016, 13671370 [DOI: 10.1109/LPT.2016.2542099].

[2] Chorchos Ł., Turkiewicz J.P.: Experimental performance of semiconductor optical amplifiers and praseodymium-doped fiber amplifiers in 1310-nm dense wavelength division multiplexing system. Optical Engineering 56/2017, 046101 [DOI 10.1117/1.OE.56.4.046101].

[3] Czyżak P., Mazurek P., Turkiewicz J.P.: $1310 \mathrm{~nm}$ Raman amplifier utilizing high-power, quantum-dot pumping lasers. Optics \& Laser Technology, 64/2014, 195-203 [DOI: 10.1016/j.optlastec.2014.05.013].

[4] https://www.nobelprize.org/prizes/physics/2009/kao/facts/ (available 30.05.2019).

[5] Kropp J.-R., Steinle G., Schäfer G., Shchukin V.A., Ledentsov N.N., Turkiewicz J.P., Zoldak M.: Accelerated aging of $28 \mathrm{~Gb} \mathrm{~s}^{-1} 850 \mathrm{~nm}$ vertical-cavity surfaceemitting laser with multiple thick oxide apertures. Semiconductor Science and Technology 30/2015 [DOI: 10.1088/0268-1242/30/4/045001].

[6] Larrode M.G., Koonen A.M.J., Vegas-Olmos J.J., Verdurmen E.J.M., Turkiewicz J.P.: Dispersion tolerant radio-over-fibre transmission of 16 and 64 QAM radio signals at $40 \mathrm{GHz}$. Electronics Letters 42/2006, 872-874 [DOI: 10.1049/el:20061311].

[7] Ledentsov N.N., Shchukin V.A., Kalosha V.P., Ledentsov N.N., Kropp J.-R., Agustin M., Chorchos Ł., Stępniak G., Turkiewicz J.P., Shi J.-W: Antiwaveguiding vertical-cavity surface-emitting laser at $850 \mathrm{~nm}$ : From concept to advances in high-speed data transmission. Optics Express 26/2018, 445-453 [DOI 10.1364/OE.26.000445].

[8] Markowski K., Chorchos L., Turkiewicz J.P.: Influence of the Four-Wave Mixing in short and medium range $1310 \mathrm{~nm}$ DWDM systems. Applied Optics 55/2016, 3051-3057 [DOI 10.1364/AO.55.003051].

[9] Mazurek P., Czyżak P., de Waard, H., Turkiewicz J.P.: Up to 112 Gbit/s single wavelength channel transmission in the $1310 \mathrm{~nm}$ wavelength domain. Microwave and Optical Technology Letters 2/2014, 263-265 [DOI: $10.1002 / \mathrm{mop} .28054]$.

[10] Mazurek P., Czyżak P., de Waardt H., Turkiewicz J.P.: SOA and Raman amplification for $1310 \mathrm{~nm}$ DWDM transmission. Optical Engineering 54/2015, 116104 [DOI: 10.1117/1.OE.54.11.116104].

[11] Mazurek P., de Waardt H., Turkiewicz J.P.: Towards 1 Tbit/s SOA based 1310 $\mathrm{nm}$ transmission for LAN/data center applications. IET Optoelectronics 9/2015, 1-9 [DOI: 10.1049/iet-opt.2014.0031]
[12] Nesset D., Wright P.: Raman extended GPON using $1240 \mathrm{~nm}$ semiconductor quantum-dot lasers. Optical Fiber Communication Conference (OFC) 2010, OThW6, April 2010.

[13] Puerta R., V. Olmos, J.J., Tafur Monroy I., Ledentsov N.N., Turkiewicz J.P.: Flexible MultiCAP Modulation and its Application to $850 \mathrm{~nm}$ VCSEL-MMF Links. IEEE Journal of Lightwave Technology 35/2017, 3168-3173 [DOI: 10.1109/JLT.2017.2701887].

[14] Puerta Ramirez R., Agustin M., Chorchos L., Toński J., Kropp J.R., Ledentsov Jr. N., Shchukin V.A.,. Ledentsov N.N, Henker R., Tafur Monroy I., Vegas Olmos J.J., Turkiewicz J.P.: Effective $100 \mathrm{~Gb} / \mathrm{s} \mathrm{IM} / \mathrm{DD} 850 \mathrm{~nm}$ multi- and single-mode VCSEL transmission through OM4 MMF. IEEE Journal of Lightwave Technology 35/2017, 423-429 [DOI: 10.1109/JLT.2016.2625799].

[15] Recommendations ITU-T G.652 Characteristics of a Single-Mode Optical Fiber and Cable, (10/2009). Online. http://www.itu.int/rec/T-RECG.652-200911-I.

[16] Stępniak G., Chorchos Ł., Agustin M., Kropp J.-R., Ledentsov N.N., Shchukin V.A, Ledentsov Jr. N.N., Turkiewicz J.P.: Up to $108 \mathrm{~Gb} / \mathrm{s}$ PAM $850 \mathrm{~nm}$ Multi and Single Mode VCSEL Transmission over $100 \mathrm{~m}$ of Multi Mode Fiber. ECOC 2016; 42nd European Conference on Optical Communication, Dusseldorf, Germany, 2016, 1-3.

[17] Stepniak G., Lewandowski A., Kropp J.R., Ledentsov N.N., Shchukin V.A., Ledentsov Jr. N., Schaefer G., Agustin M., Turkiewicz J.P.: 54 Gbps OOK transmission using single mode VCSEL up to $2.2 \mathrm{~km}$ MMF. IET Electronics Letters 52/2016, 633-635 [DOI: 10.1049/el.2015.4264]

[18] Thipparapu N.K., Umnikov A.A., Barua P., Sahu J.K.: Bi-doped fiber amplifier with a flat gain of $25 \mathrm{~dB}$ operating in the wavelength band $1320-1360 \mathrm{~nm}$. Optics Letters 41/2016, 1518-1521 [DOI: 10.1364/OL.41.001518].

[19] Turkiewicz J.P., Kropp J.-R., Ledentsov N.N., Shchukin V.A., Schafer G.: High speed optical data transmission with compact $850 \mathrm{~nm}$ TO-can assemblies. IEEE Journal of Quantum Electronics 50/2014, 281-286 [DOI 10.1109/JQE.2014.2304742]

[20] Turkiewicz J.P., Tangdiongga E., Rohde H., Schairer W., Lehmann G., Khoe G.D., de Waardt H.: Simultaneous high speed OTDM add-drop multiplexing using GT-UNI switch. Electronics Letters 10/2003, 795-796 [DOI: 10.1049/el:20030535].

[21] Turkiewicz J.P., de Waardt H.: Low Complexity up to 400-Gb/s Transmission in the $1310 \mathrm{~nm}$ Wavelength Domain. IEEE Photonics Technology Letters 11/2012, 942-944 [DOI: 10.1109/LPT.2012.2191278].

[22] Turkiewicz J.P., Khoe G.D., de Waardt H.: All-optical 1310 to $1550 \mathrm{~nm}$ wavelength conversion by utilising nonlinear polarisation rotation in semiconductor optical amplifier. Electronics Letters 1/2005, 29-30 [DOI: 10.1049/el:20057435].

[23] Turkiewicz J.P.: Cost-effective $\mathrm{n} \times 25 \mathrm{Gbit} / \mathrm{s}$ DWDM transmission in the $1310 \mathrm{~nm}$ wavelength domain. Optical Fiber Technology 17/2011, 179-184 [DOI 10.1016/j.yofte.2011.01.010].

[24] Turkiewicz J.P: Analysis of the SSMF zero-dispersion wavelength location and its influence on high capacity $1310 \mathrm{~nm}$ transmission. 2013 Optical Fiber Communication Conference and Exposition and the National Fiber Optic Engineers Conference (OFC/NFOEC), Anaheim, CA, 2013, 1-3 [DOI: 10.1364/NFOEC.2013.JW2A.06].

[25] Wieckowski M., Jensen J.B., Tafur Monroy I., Siuzdak J., Turkiewicz J.P.: $300 \mathrm{Mbps}$ transmission with $4.6 \mathrm{bit} / \mathrm{s} / \mathrm{Hz}$ spectral efficiency over $50 \mathrm{~m}$ PMMA POF link using RC-LED and multilevel Carrierless Amplitude Phase modulation. 2011 Optical Fiber Communication Conference and Exposition and the National Fiber Optic Engineers Conference, Los Angeles, CA, 2011, 1-3.

[26] Winzer P. J.: Energy-efficient optical transport capacity scaling through spatial multiplexing. IEEE Photonics Technology Letters, 23/2011, 851-853 [DOI 10.1109/LPT.2011.2140103].

\section{Ph.D. Jarosław Piotr Turkiewicz}

e-mail: jturkiew@tele.pw.edu.pl

J.P. Turkiewicz received Ph.D. degree in optica communication from the Eindhoven University of Technology, The Netherlands in 2006 and D.Sc. in 2014 from the Warsaw University of Technology. Since 2007 he is with Institute of Telecommunication, Warsaw University of Technology. He published over 100 peer reviewed papers as well as contributed and led several national and international research projects. His scientific interests include high speed optical signal transmission and switching.

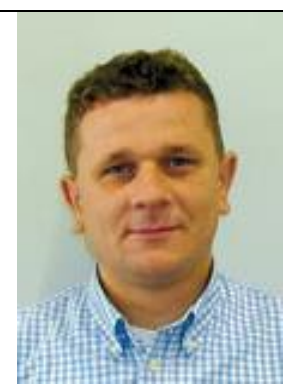

ORCID ID: 0000-0003-2345-4147

otrzymano/received: 15.05.2019 przyjęto do druku/accepted: 15.06 .2019 